\title{
From stem cells to red blood cells: how far away from the clinical application?
}

\author{
XIE XiaoYan ${ }^{1,2 \dagger}$, LI YanHua ${ }^{1,2 \dagger} \&$ PEI XueTao ${ }^{1,2 *}$ \\ ${ }^{1}$ Stem Cell and Regenerative Medicine Lab, Beijing Institute of Transfusion Medicine, Beijing 100850, China; \\ ${ }^{2}$ South China Research Center for Stem Cell and Regenerative Medicine, Guangzhou 510005, China
}

Received February 15, 2014; accepted April 5, 2014; published online May 13, 2014

\begin{abstract}
The generation of red blood cells (RBCs) from stem cells provides a solution for deficiencies in blood transfusion. Currently, primary hematopoietic stem cells, embryonic stem cells and induced pluripotent stem cells have shown the potential to produce fully mature RBCs. Here, we discuss the advantages, induction protocols, progress and possible clinical applications of stem cells in RBC production.
\end{abstract}

stem cells, red blood cells, differentiation, clinical application

Citation: Xie XY, Li YH, Pei XT. From stem cells to red blood cells: how far away from the clinical application?. Sci China Life Sci, 2014, 57: 581-585, doi: $10.1007 / \mathrm{s} 11427-014-4667-5$

The shortage of blood for transfusions is a common problem in developing countries. Since 2010, numerous media outlets have reported deficiencies with regards to blood storage options in China, especially for rare blood type antigens. For the moment, blood donations are the only source for transfusions. Considering the insufficient numbers of blood donors, and possible contamination with undetectable pathogens, it is urgent that alternative blood sources for transfusions are found. Stem cells are a possible alternative source to generate red blood cells (RBCs).

Stem cells are undifferentiated cells that can differentiate into specialized cells and divide to produce more cells. All categorized stem cells, including hematopoietic stem cells (HSCs, a type of adult stem cell), embryonic stem cells (ESCs) and induced pluripotent stem cells (iPSCs), have the ability to differentiate into fully mature RBCs. However, the clinical application of stem cells for producing RBCs that can be used in transfusions requires further development. In

$\dagger$ Contributed equally to this work

*Corresponding author (email: peixt@nic.bmi.ac.cn) this review, we discuss the milestones and common protocols for inducing various stem cells in RBCs, the advantages and disadvantages of these cell resources, and the potential clinical application of these cells.

\section{HSC-derived RBCs}

The first identified stem cells were HSCs; they possess the potential to differentiate into every blood cell type, including RBCs. The transplantation of HSCs is now routinely used in the treatment of diverse malignant and non-malignant diseases to replace or rebuild a patient's hematopoietic system [1]. HSCs can be isolated from bone marrow, peripheral blood or cord blood. Using CD34 as a marker of human HSCs, there are around 1\%-5\% HSCs in bone marrow nucleated cells, $1 \%$ in cord blood cells, and less than $0.1 \%$ HSCs in normal peripheral blood cells.

Typically, the development of HSCs into RBCs involves different stages including common myeloid progenitors (CMPs), megakaryocyte-erythroid progenitors (MEPs), 
burst forming unit-erythroid (BFU-E), colony forming unit-erythroid (CFU-E), proerythroblasts (ProE), erythroblasts/basophilic normoblasts (BasoE), polychromatophilic normoblasts (PolyE), orthochromatic normoblasts (OrthoE), reticulocytes (Retic), and mature RBCs (Figure 1). The maturation of RBCs usually takes place in the bone marrow as this microenvironment provides a niche for HSCs. The in vitro production of mature RBCs could be fulfilled by simulating the HSC niche in vivo. Using optimized media, combinations of cytokines, and stromal cells, researchers have successfully expanded and induced erythrocytes from HSCs. Neildez-Nquyen et al. reported that sequential application of specific growth factor combinations in serum-free culture medium resulted in erythroid precursors that could fully mature when transplanted into immunodeficient mice [2]. These immature blood cells could be used for the treatment of chronic anemia and in gene therapy; however, they are not fully functional immediately after transfusion. Giarratana et al. [3] demonstrated the application of cytokines and co-culture on stromal cells for large-scale ex vivo production of mature human RBCs from HSCs of diverse origins. Enucleated RBCs meet the demand of functional blood cells during acute hemorrhaging; however, the dependency on stromal cells hampers the application of this method. Miharadac et al. [4] attempted to develop an in vitro stromal cell-independent model for producing RBCs. Despite this progress, the generation of large-scale, fully mature, and clinically applicable RBCs remains an obstacle to researchers.

The in vitro induction and expansion of RBCs from HSCs can be divided into three steps: erythroid lineage determination, expansion of erythroid progenitors, and RBC maturation [5]. In some instances an extra step for HSC amplification is necessary beforehand. Cytokines and supplements applied to promote erythroid progenitor proliferation include lipids, SCF, IL-3, EPO, and/or a glucocorticoid receptor (GR) agonist. Reports trying to improve the expansion and differentiation of erythroid progenitors showed that chromatin-modifying agents (such as valproic acid), hypoxia, agents that stabilize the transcription factor HIF- $1 \alpha$, and steroid hormones have a role during these processes [6-10].
For the maturation of erythrocytes, EPO and IGF-I are required. However, regulators for erythrocyte enucleation remain to be identified. It was suggested that no cytokines other than stromal cells or matrix are necessary for enucleation [11] (Figure 1). The establishment of massive in vitro expansion methods for human RBCs is a prerequisite for the clinical application of these cells; therefore, greater effort should be applied to achieve higher proliferation capacities and greater specificity of fully mature RBCs.

Although RBCs generated ex vivo are not in as much demand for transfusion, appropriate animal models and proof-of-principle human transfusion studies have confirmed their function in vivo. Immunodeficient mice were first used to evaluate the in vivo function of cultured RBCs. $\mathrm{Hu}$ and coworkers further suggested that by depleting macrophages, human RBCs could develop and function better in immunodeficient mice [12]. Primates are also excellent animal models based on their close relationship to humans. In 2011, Giarratana and colleagues produced the first transplantable RBCs ex vivo [13]. In their study, $10^{10}$ RBCs were generated from peripheral CD $34^{+}$HSCs and transplanted into a health volunteer. These RBCs survived more than 26 $\mathrm{d}$ in the circulatory system, verifying their quality and function in vivo.

Increasing knowledge of hematopoiesis and erythropoiesis makes the generation of RBCs from HSCs an accessible goal. However, the limited proliferation ability of HSCs restricts the usage of these cells. The high cost of cell culture is another obstacle that needs to be overcome before these cells can be applied in a clinical setting.

\section{Pluripotent stem cell-derived HSCs and RBCs}

Currently, HSCs used during clinical transplantation are usually derived from cord blood, adult bone marrow or peripheral blood. Given the shortage of donors and limited supply of HSCs, human ESCs have become an alternative source of HSCs and mature blood cells for therapeutic purposes. ESCs have the capacity for nearly unlimited prolifer-

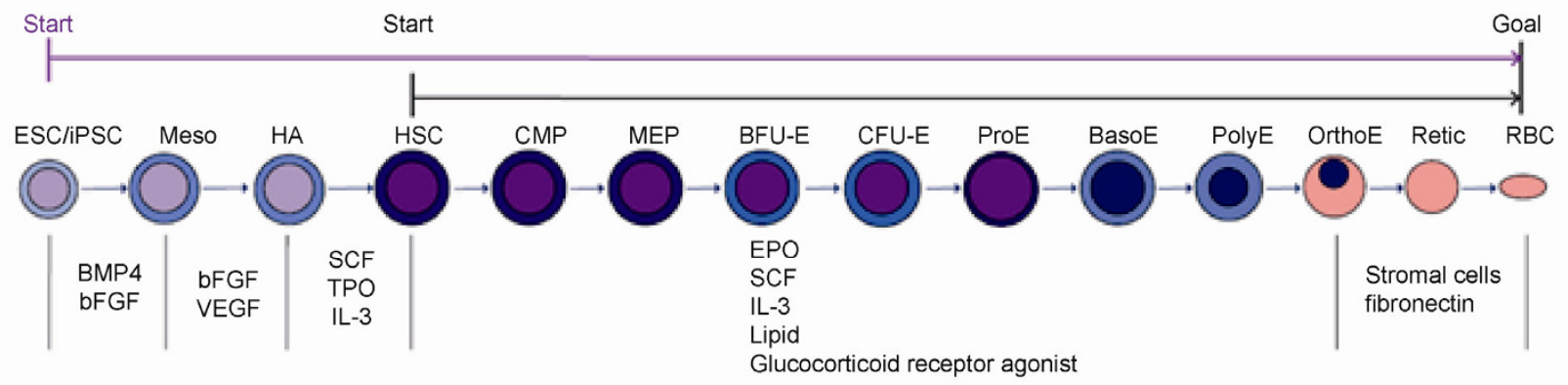

Figure 1 Differentiation of pluripotent stem cells into RBCs. Meso, mesoderm; HA, hemangioblast; CMP, common myeloid progenitor; MEP, megakaryocyte-erythroid progenitor; BFU-E, burst forming unit-erythroid; CFU-E, colony forming unit-erythroid; ProE, proerythroblast; BasoE, basophilic erythroblast; PolyE, polychromatophilic erythroblast; OrthoE, orthochromatic erythroblast; Retic, reticulocyte. 
ation and can differentiate into cells/tissues of all three germ layers. In recent years, induced pluripotent stem cells (iPSCs) with the characteristics of ESCs have been obtained using adult somatic cell reprogramming, which involves the overexpression of key transcription factors [14], or culturing with combinations of exogenous small molecules [15]. The development of iPSC technology has revolutionized the possibilities of personalized cell therapy. These iPSCs could serve as an embryo-free source of patient-specific stem cells with reduced human leucocyte antigen (HLA) mismatching. Taylor suggested that a tissue bank from 150 selected homozygous HLA-typed volunteers could match $93 \%$ of the UK population with a minimal requirement for immunosuppression [16]. For RBC transfusion, nucleated cells are routinely removed from RBC concentrates, while RBCs minimally express HLA molecules. Therefore, pluripotent stem cells such as ESCs and iPSCs are able to create RBCs with universal $\mathrm{O}$ and rhesus (RhD)-negative blood types. The use of iPSC technology could also be applied to modify the mutated genes from patients with inherited RBC disorders, such as sickle cell anemia [17] and $\alpha$-thalassemia [18]. Several groups have generated gene-corrected $\beta$-thalassemia iPSCs from patients; these could be induced to differentiate into hematopoietic progenitor cells and then into erythroblasts expressing normal $\beta$-globin [19-21].

It has been reported that pluripotent stem cells can be induced to differentiate into hematopoietic cells using three different culture methods: embryoid body formation; stromal cell-based co-culturing; and monolayer culturing [22-24]. These different induction strategies often engage several sets of cytokines to provide a simulated microenvironment for hematopoiesis. Pluripotent stem cells undergo several stages when differentiating into hematopoietic cells (Figure 1). Bone morphogenetic protein-4 (BMP-4) and basic fibroblast growth factor (bFGF) were employed to induce the differentiation of mesoderm progenitors [25]. Vascular endothelial growth factor (VEGF) and bFGF were used to promote hemangioblast specification [26]. Hematopoietic cytokines, such as stem cell factor (SCF), Fms-like tyrosine kinase 3 (Flt-3), interleukin-3 (IL-3), IL-6, thrombopoietin (TPO) and erythropoietin (EPO), were used to promote the formation of hematopoietic cells. Until now, the generation efficiency of $\mathrm{CD} 34^{+}$hematopoietic cells from pluripotent stem cells has required improvement. A greater understanding of hematopoiesis mechanisms might help us develop more efficient induction strategies for hematopoietic cells. McKinney-Freeman et al applied a network biology-based analysis to reconstruct the gene regulatory networks during sequential stages of HSC development [27]. They found that HSCs from in vitro differentiated embryonic stem cells closely resembled definitive HSCs; however, they lacked a Notch signaling signature. Their results suggested that an exogenous Notch ligand could be added to the induction system and facilitate hematopoietic commitment [27]. Delaney reported that the Notch ligand can pro- mote the expansion of cord blood HSCs [28]. Although in vitro culture methods for the differentiation of pluripotent stem cells into hematopoietic cells have been improved, optimization is required to obtain HSCs that are more suitable for transplantation. To efficiently generate engrafted hematopoietic cells, forced expression of certain transcription factors such as HOXB4 and RUNX1a is an alternative approach [29,30]. Recently, Daley's lab reported a strategy to re-specify $\mathrm{CD} 34^{+} \mathrm{CD} 45^{+}$myeloid precursors from pluripotent stem cells into multi-lineage progenitors that can be expanded in vitro and engrafted in vivo using five transcription factors [31]. These engrafted human erythroblasts from pluripotent stem cells underwent hemoglobin switching in vivo.

Pluripotent stem cells have been shown to differentiate into an erythroid population [32]. These erythroid cells began to express $\beta$-globin and approximately $40 \%$ of the RBCs became enucleated [33]. These differentiated erythroid cells showed similar functions as RBCs derived from cord blood. However, scalable expansion and generation of mature erythrocytes from pluripotent stem cells remains a problem. Recently, Hirose reported that immortalization of erythroblasts by overexpression of c-MYC and BCL-XL enabled large-scale erythrocyte production from human pluripotent stem cells [34]. Another group demonstrated the generation of large-scale human ESCs into functional erythrocytes using a sequential four-step procedure [35]. They reported that $10^{11}-10^{12}$ nucleated erythroid cells can be produced from approximately $10^{7}$ human ESCs; their differentiated RBCs had comparable functions with normal adult RBCs. However, full maturation and efficient enucleation of erythroid cells from pluripotent stem cells are yet to be accomplished. A deeper understanding of the process of erythrocyte maturation would be helpful in directing the $e x$ vivo generation of functionally mature RBCs from stem cells.

\section{Future perspectives}

Progress has been made over the last 10 years in the generation of a large number of RBCs from stem cells ex vivo. However, there are several issues that remain to be resolved. Over the next 5-10 years, much work needs to be conducted surrounding the efficient differentiation of erythroid cells from stem cells, the switch to adult $\beta$-globin, and enucleation of erythrocytes. Given the limited number of HSCs derived from cord blood or adult sources, large-scale manufacturing of RBCs from pluripotent stem cells will be required. However, there remain challenges in the production of a large number of RBCs that can be used clinically. Appropriate bioreactor design and culture will be required to realize RBC manufacturing for clinical applications; the cost of RBC products is another consideration. Biosafety is particularly important for the application of RBCs generated 
ex vivo, with good manufacturing practices needed to produce stem cell-derived RBCs. This should encompass the choice of stem cells, source of reagents, the use of defined component-free animal culture environments, quality control, and quality assurance procedures. Novel RBC products from stem cells are highly suitable as an alternative to blood transfusion. If the barrier of obtaining enough RBCs from stem cells is resolved, these cells might become the first stem cell-derived products to be reliably used in a clinical setting.

This work was supported by the National High Technology Research and Development Program of China (2013AA020107), National Basic Research Program of China (2011CB964804) and National Natural Science Foundation of China (31101040).

1 Kondo M, Wagers AJ, Manz MG, Prohaska SS, Scherer DC, Beilhack GF, Shizuru JA, Weissman IL. Biology of hematopoietic stem cells and progenitors: implications for clinical application. Annu Rev Immunol, 2003, 21: 759-806

2 Neildez-Nguyen TMA, Wajcman H, Marden MC, Bensidhoum M, Moncollin V, Giarratana M-C, Kobari L, Thierry D, Douay L. Human erythroid cells produced ex vivo at large scale differentiate into red blood cells in vivo. Nat Biotech, 2002, 20: 467-472

3 Giarratana MC, Kobari L, Lapillonne H, Chalmers D, Kiger L, Cynober T, Marden MC, Wajcman H, Douay L. Ex vivo generation of fully mature human red blood cells from hematopoietic stem cells. Nat Biotech, 2005, 23: 69-74

4 Miharada K, Hiroyama T, Sudo K, Nagasawa T, Nakamura Y. Efficient enucleation of erythroblasts differentiated in vitro from hematopoietic stem and progenitor cells. Nat Biotech, 2006, 24: 1255-1256

5 Zeuner A, Martelli F, Vaglio S, Federici G, Whitsett C, Migliaccio AR. Concise review: stem cell-derived erythrocytes as upcoming players in blood transfusion. Stem Cells, 2012, 30: 1587-1596

6 Migliaccio G, Sanchez M, Masiello F, Tirelli V, Varricchio L, Whitsett C, Migliaccio AR. Humanized culture medium for clinical expansion of human erythroblasts. Cell Transplant, 2010, 19: 453-469

7 Chaurasia P, Berenzon D, Hoffman R. Chromatin-modifying agents promote the ex vivo production of functional human erythroid progenitor cells. Blood, 2011, 117: 4632-4641

8 Leberbauer C, Boulmé F, Unfried G, Huber J, Beug H, Müllner EW. Different steroids co-regulate long-term expansion versus terminal differentiation in primary human erythroid progenitors. Blood, 2005, 105: 85-94

9 Narla A, Dutt S, McAuley JR, Al-Shahrour F, Hurst S, McConkey M, Neuberg D, Ebert BL. Dexamethasone and lenalidomide have distinct functional effects on erythropoiesis. Blood, 2011, 118: 2296-2304

10 Flygare J, Estrada VR, Shin C, Gupta S, Lodish HF. Hif1 $\alpha$ synergizes with glucocorticoids to promote BFU-E progenitor self-renewal. Blood, 2011, 117: 3435-3444

11 Keerthivasan G, Wickrema A, Crispino JD. Erythroblast enucleation. Stem Cells Int, 2011, 2011: 9

12 Hu Z, Van Rooijen N, Yang YG. Macrophages prevent human red blood cell reconstitution in immunodeficient mice. Blood, 2011, 118: 5938-5946

13 Giarratana MC, Rouard H, Dumont A, Kiger L, Safeukui I, Le Pennec PY, Francois S, Trugnan G, Peyrard T, Marie T, Jolly S, Hebert N, Mazurier C, Mario N, Harmand L, Lapillonne H, Devaux JY, Douay L. Proof of principle for transfusion of in vitro generated red blood cells. Blood, 2011, 118: 5071-5079

14 Takahashi K, Tanabe K, Ohnuki M, Narita M, Ichisak T, Tomoda K, Yamanaka S. Induction of pluripotent stem cells from adult human fibroblasts by defined factors. Cell , 2007, 131: 861-872

15 Hou P, Li Y, Zhang X, Liu C, Guan J, Li H, Zhao T, Ye J, Yang W, Liu K, Ge J, Xu J, Zhang Q, Zhao Y, Deng H. Pluripotent stem cells induced from mouse somatic cells by small-molecule compounds. Science, 2013, 341: 651-654

16 Taylor CJ, Peacock S, Chaudhry AN, Bradley JA, Bolton EM. Generating an iPSC bank for HLA-matched tissue transplantation based on known donor and recipient HLA types. Cell Stem Cell, 2012, 11: $147-152$

17 Zou J, Mali P, Huang X, Dowey SN, Cheng L. Site-specific gene correction of a point mutation in human iPS cells derived from an adult patient with sickle cell disease. Blood, 2011, 118: 4599-4608

18 Chang CJ, Bouhassira EE. Zinc-finger nuclease-mediated correction of $\alpha$-thalassemia in iPS cells. Blood, 2012, 120: 3906-3914

19 Wang Y, Zheng CG, Jiang Y, Zhang J, Chen J, Yao C, Zhao Q, Liu S, Chen K, Du J, Yang Z, Gao S. Genetic correction of $\beta$-thalassemia patient-specific iPS cells and its use in improving hemoglobin production in irradiated SCID mice. Cell Res, 2012, 22: 637-648

20 Fan Y, Luo Y, Chen X, Li Q, Sun X. Generation of human $\beta$-thalassemiainduced pluripotent stem cells from amniotic fluid cells using a single excisable lentiviral stem cell cassette. J Reprod Dev, 2012, 58: 404-409

21 Ma N, Liao B, Zhang H, Wang L, Shan Y, Xue Y, Huang K, Chen S, Zhou X, Chen Y, Pei D, Pan G. Transcription activator-like effector nuclease (TALEN)-mediated gene correction in integration-free $\beta$-thalassemia induced pluripotent stem cells. J Biol Chem, 2013, 288: 34671-34679

22 Dang SM, Kyba M, Perlingeiro R, Daley GQ, Zandstra PW. Efficiency of embryoid body formation and hematopoietic development from embryonic stem cells in different culture systems. Biotechnol Bioeng, 2002, 78: 442-453

23 Vodyanik MA, Bork JA, Thomson JA, Slukvin II. Human embryonic stem cell-derived $\mathrm{CD} 34^{+}$cells: efficient production in the coculture with OP9 stromal cells and analysis of lymphohematopoietic potential. Blood, 2005, 105: 617-626

24 Niwa A, Heike T, Umeda K, Oshima K, Kato I, Sakai H, Suemori H, Nakahata T, Saito MK. A novel serum-free monolayer culture for orderly hematopoietic differentiation of human pluripotent cells via mesodermal progenitors. PLoS ONE, 2011, 6: e22261

25 Bernardo AS, Faial T, Gardner L, Niakan KK, Ortmann D, Senner CE, Callery EM, Trotter MW, Hemberger M, Smith JC, Bardwell L, Moffett A, Pedersen RA. BRACHYURY and CDX2 mediate BMP-induced differentiation of human and mouse pluripotent stem cells into embryonic and extraembryonic lineages. Cell Stem Cell, 2011, 9: 144-155

26 Wang C, Tang X, Sun X, Miao Z, Lv Y, Yang Y, Zhang H, Zhang P, Liu Y, Du L, Gao Y, Yin M, Ding M, Deng H. TGFß inhibition enhances the generation of hematopoietic progenitors from human ES cell-derived hemogenic endothelial cells using a stepwise strategy. Cell Res, 2012, 22: 194-207

27 McKinney-Freeman S, Cahan P, Li H, Lacadie SA, Huang HT, Curran M, Loewer S, Naveiras O, Kathrein KL, Konantz M, Langdon EM, Lengerke C, Zon LI, Collins JJ, Daley GQ. The transcriptional landscape of hematopoietic stem cell ontogeny. Cell Stem Cell, 2012, 11: 701-714

28 Delaney C, Heimfeld S, Brashem-Stein C, Voorhies H, Manger RL, Bernstein ID. Notch-mediated expansion of human cord blood progenitor cells capable of rapid myeloid reconstitution Nat Med, 2010, 16: $232-236$

29 Bowles KM, Vallier L, Smith JR, Alexander MR, Pedersen RA. HOXB4 overexpression promotes hematopoietic development by human embryonic stem cells. Stem Cells, 2006, 24: 1359-1369

30 Ran D, Shia WJ, Lo MC, Fan JB, Knorr DA, Ferrell PI, Ye Z, Yan M, Cheng L, Kaufman DS, Zhang DE. RUNX1a enhances hematopoietic lineage commitment from human embryonic stem cells and inducible pluripotent stem cells. Blood, 2013, 121: 2882-2890

31 Doulatov S, Vo LT, Chou SS, Kim PG, Arora N, Li H, Hadland BK, Bernstein ID, Collins JJ, Zon LI, Daley GQ. Induction of multipotential hematopoietic progenitors from human pluripotent stem cells via 
respecification of lineage-restricted precursors. Cell Stem Cell, 2013, 13: 459-470

32 Qiu C, Olivier EN, Velho M, Bouhassira EE. Globin switches in yolk sac-like primitive and fetal-like definitive red blood cells produced from human embryonic stem cells. Blood, 2008, 111: 2400-2408

33 Ma F, Ebihara Y, Umeda K, Sakai H, Hanada S, Zhang H, Zaike Y, Tsuchida E, Nakahata T, Nakauchi H, Tsuji K. Generation of functional erythrocytes from human embryonic stem cell-derived definitive hematopoiesis. Proc Natl Acad Sci USA, 2008, 105: $13087-13092$
34 Hirose S, Takayama N, Nakamura S, Nagasawa K, Ochi K, Hirata S, Yamazaki S, Yamaguchi T, Otsu M, Sano S, Takahashi N, Sawaguchi A, Ito M, Kato T, Nakauchi H, Eto K. Immortalization of erythroblasts by c-MYC and BCL-XL enables large-scale erythrocyte production from human pluripotent stem cells. Stem Cell Reports, 2013, 1: 499-508

35 Lu SJ, Feng Q, Park JS, Vida L, Lee BS, Strausbauch M, Wettstein PJ, Honig GR, Lanza R. Biologic properties and enucleation of red blood cells from human embryonic stem cells. Blood, 2008, 112: $4475-4484$

Open Access This article is distributed under the terms of the Creative Commons Attribution License which permits any use, distribution, and reproduction in any medium, provided the original author(s) and source are credited. 